Research Paper:

\title{
No Alternation in Treg Frequency in Peripheral Blood of Chrmical Vitems With Long-term Mild-moderate Pulmonary Complication
}

Nafiseh Zand ${ }^{1}$ D, Mahmood Bozorgmehr ${ }^{2}$ (D), Ali Reza Sabetpour ${ }^{3}$, Mohammad Mahdi Naghizadeh $^{4}$, Ensieh Sadat Mirsharif ${ }^{5}$, Tooba Ghazanfari1 ${ }^{12^{*}}$ (D)

1. Department of Immunology, School of Medicine, Shahed University, Tehran, Iran.

2. Oncopathology Research Center, Iran University of Medical Sciences, Tehran, Iran

3. Countess of Chester Hospital, Chester, UK.

4. Noncommuicable Diseases Research Center, Fasa University of Medical Sciences, Fasa, Iran.

5. Immunoregulation Research Center, Shahed University, Tehran, Iran.

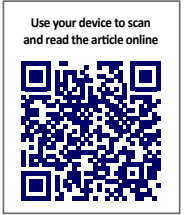

Citation Zand N, Bozorgmehr M, Sabetpour AR, Naghizadeh MM, Mirsharif ES, Ghazanfari T. No Alternation in Treg Frequency in Peripheral Blood of Chrmical Vitems With Long-term Mild-moderate Pulmonary Complication. 2022; 4(2):83-90. http://dx.doi.org/10.32598/Immunoregulation.4.2.2

doij http://dx.doi.org/10.32598/Immunoregulation.4.2.2

\section{(i) (8)}

Article info:

Received: 25 May 2021

Accepted: 22 Aug 2021

Available Online: 01 Jan 2022

Keywords:

Regulatory T cells, Sulfur mustard, Bioterrorism,

Chemical warfare

\begin{abstract}
A B S T RA C T
Background: Regulatory T Cells (Treg) are the subgroups of lymphocytes that control inflammatory responses and regulate homeostasis processes through cellular contact and the secretion of soluble agents. We investigated the frequency of Treg in peripheral blood of sulfur mustard exposed patients with CD4, CD25, CD127, FOXP3, and CD45RA markers, compared with healthy individuals and the correlation between frequency of these cells and long-term pulmonary complications.

Materials and Methods: In total, 12 sulfur mustard exposed patients and 12 healthy volunteers were invited. Clinical inspections of both groups were performed by pulmonary specialists and spyrometric evaluation that pulmonary function test operators conducted. After the isolation of peripheral blood mononuclear cells, the frequency of Treg cells was determined by flowcytometry.

Results: The frequency of Treg cells was not significantly different in those exposed to sulfur mustard. Furthermore, there was no significant correlation between spyrometric parameters and the frequency of these cells.

Conclusion: According to the current study data, there is no difference in the frequency of Treg cells between exposed patients with mild pulmonary complications and healthy volunteers. Thus, further studies are required to understand the role of these cells in the severity of pulmonary complications of these patients.
\end{abstract}

* Corresponding Author:

Tooba Ghazanfari, PhD.

Address: Department of Immunology, School of Medicine, Shahed University, Tehran, Iran.

Phone:+98 (21) 66418216

E-mail: tghazanfari@yahoo.com 


\section{Introduction}

ulfur Mustard (SM) is a chemical alkylating warfare agent, absorbed through inhalation, skin, eye, gastrointestinal tract contact, and contaminated food consumption $[1,2]$. SM was applied in the Iran-Iraq conflict and left numerous victims in Iran suffering from its adverse and inevitable effects on their quality of life. Despite international prohibitions, many new victims remain globally [3-5]. SM exposure results in both acute and chronic inflammatory complications. Early complications appear in the first week and delayed complications 10 to 15 years later, even after long years. Symptoms manifest by various ocular, cutaneous and pulmonary $[6,7]$.

The respiratory disorder is a prevalent problem among SM-affected people. According to Iran Sardasht's study, approximately $80 \%$ of exposed people about $16-20$ years after mustard gas exposure suffered from chronic Chronic Obstructive Pulmonary Diseases (COPD) symptoms (including cough, phlegm, chest pain, \& dyspnea) [6-10]. Moreover, SM has a carcinogenic character and may raise the risk of lung cancer in SM exposed patients [11]. However, the mechanism of chronic injury remains unestablished. Different studies demonstrated that dysregulated immune system response might contribute to uncontrolled inflammation in these patients as a most threatening problem. Indeed, change in proinflammatory and anti-inflammatory cytokines such as TGf- $\beta$, IL-6, IL-17, IL-8, IL-1 $\beta$, IL-12 [Tumor growth factor (TGF $\beta$ ), Interlukine (IL)-g], and the frequency of some immune cells such as CTL, CD4+Th1, Th2, Th17 [cytoxic t cell (CTL), CD4+T helper (Th)1] supported this statement [12-17].

Treg cells are subgroups of $\mathrm{T}$ cells that play a crucial role in maintaining immune homeostasis by suppressing and regulating cytokine secretion and proliferation of other cell types by different mechanisms [18, 19]. Various studies focused on Treg Impairment in the context of chronic inflammatory diseases such as COPD and autoimmune diseases [20]. Additionally, they attempted to manipulate their impairment and develop therapies for inflammation regulation, which have addressed the possible relation between chronic inflammation and Treg frequency in sulfur mustard patients. The frequency of Treg in other studies was evaluated and indicated an increase. Still, Treg is only characterized by staining for CD4 and FOXP3 markers because of the transitory expression of FOXP3 by other T cells. Not only might distinguishing Treg from other $\mathrm{T}$ cells be difficult but the Treg fraction might also be underestimated [21]. However, the definition of Treg is controversial in various studies. We assessed the Treg cells' frequency for the first time with the most common combinations of markers such as CD4, CD25, CD127, FOXP3, and CD45RA; Whish seems to subgroup Treg based on activation status.

\section{Materials and Methods}

Overall, 12 patients were selected from the male individuals exposed to SM in the Iran-Iraq war, and An experienced pulmonologist examined respiratory signs and symptoms. All paitents presented mild to moderate pulmonary involvement based on the Global initiative for chronic Obstructive Lung Disease (GOLD).12 sexmatched control subjects were enrolled in this study too. All the study subjects' ages ranged from 35-65 years. The study participants were ineligible or excluded from the study if they had any of the following conditions: 1) acute or chronic infection, 2) recent treatment with immuno-suppressive drugs, 3 ) age $<35$ and $>65$ years, 4 ), and a history of smoking.

This study was approved by the Local Ethics Committee of Shahed University (Shahed.REC.13961218). Before participation, informed consent was provided from all the subjects involved in the study.

\section{Lung function tests}

Spyrometry was performed in all patients according to the American Thoracic Society under the supervision of a trained nurse before the blood samples collection [22]. The explored patients were seated on a comfortable chair, received standardized instructions from the nurse, and requested forced expiratory maneuvers. The Forced Vital Capacity (FVC) and Forced Expiratory Volume in the first second (FEV1) were measured three times, and the best recordings were selected.

\section{Sample preparation}

In total, $2.5 \mathrm{~mL}$ peripheral blood samples from each subject were collected in Ethylene Diamine Tetra Acetic Acid (EDTA)-treated tubes. Peripheral Blood Mononuclear Cells (PBMCs) were isolated according to milteny Biotec PBMC isolation protocol with slight differences in using Ficoll (Lymphodex; Inno-Train Diagnostik, Kronberg, Germany) density gradient centrifugation. Blood samples were diluted 1:2 with PBS, layered onto Ficoll, and centrifuged at $400 \mathrm{~g}$ for $30 \mathrm{~min}$ at room temperature. The PBMC layer was collected, washed, and centrifuged at $300 \mathrm{~g}$ for $10 \mathrm{~min}$ at $4{ }^{\circ} \mathrm{C}$. Next, the cells 
were centrifuged at $200 \mathrm{~g}$ for $10 \mathrm{~min}$ at $4{ }^{\circ} \mathrm{C}$, and viable counts were obtained using Tryrpan blue.

\section{Flow cytometry analysis}

To detect Tregs using flow cytometry, the following monoclonal antibodies were used: anti- CD4-Alexaflour 700 (clone L200), anti- CD25-APC (clone M-A251), anti-CD45RA-Percp cy5.5 (cloneHI100), anti-CD127BB515 (cloneHIL-7R-M21), anti-FOXP3-PE (clone 259D/C7), all monoclonal antibodies were purchased from BD Biosciences (San Jose, CA, USA). Before use, all Abs were titrated to find the proper antibody concentration. Immuostaining was performed according to the manufacturer's instructions.

Briefly, to exclude dead cells, $1 \mu \mathrm{L}$ fixable viability stain 780 (BD Biosciences, San Diego, CA) was added to 106 PBMCs after two steps of washing cells, and the cells were labeled at $4{ }^{\circ} \mathrm{C}$ for $30 \mathrm{~min}$ with the antibodies for surface markers; subsequently, the cells were washed twice and resuspended in staining buffer (PBS-FBS 2\% [Phosphate Buffered Saline with 2\% Fetal Bovine Serum]). For Intracellular staining, the cells were then fixed and permeabilized using a transcription buffer set (BD Biosciences, San Diego, CA) washed two times with BD Biosciences Permeabilization buffer, and incubated with anti- FOXP3-PE at $4^{\circ} \mathrm{C}$ for $30 \mathrm{~min}$ until sample measurement using the flow cytometer. Fluorescence Minus One (FMO) controls for CD25 and CD45RA were used to distinguish the positive populations. To accurately compensate spectral overlap across channels. Compensation controls for each fluorochrom were prepared using Onecomp ebeads (ebioscience, USA).

Data were acquired on an Attune nxt flow cytometer (ThermoFisher, USA) and were analyzed using the Flowjo software vX (Tree Star, Ashland, OR). The gating strategy for detecting Treg cells and their subsets is presented in Figure 1.

The Treg cells' absolute count was calculated by multiplying the frequency of interest obtained using flow cytometry by the lymphocyte count in Cell Blood Count (CBC) report prepared on the hematology analyzer (Sysmex XS-500i, Hyogo, Japan) divided by 100.

SPSS was used to analyze the acquired data. As a nonparametric statistical test, Spearman's rank correlation coefficient test was applied for potential associations between Spyrometric parameter and Treg frequency. Independent Student's t-test was used to determine significant differences between the variables as a para- metric test. Results were expressed as mean plus-minus Standard Deviation (Mean \pm SD). Statistical significance difference was considered $\mathrm{P}<0.05$.

\section{Results}

This study investigated the frequency of Treg in sulfur mustard exposed patients and compared it with that of healthy volunteers. To determine the absolute count of Treg, we first measured the WBC and differential (i.e., platelet, neutrophil, lymphocyte, monocyte, eosinophil) counts; however, found no significant differences between them (Table 1).

Next, we performed immuno phenotypying with flowcytometry (Table 2). There was no significant difference between $\mathrm{CD} 4+$ cell percentage and absolute numbers among lymphocytes $(\mathrm{P}=1.126, \mathrm{P}=1.825$, respectively).

Due to the difficulty of Treg description, we evaluate the common definition of Treg phenotype, CD4+CD25+CD127- is most common, and percentage and absolute numbers of it didn't vary between sulfur mustard exposed healthy volunteer $(\mathrm{P}=1.192, \mathrm{P}=1.292$, respectively). FOXP3 as an essential marker was also evaluated in the panel of Treg makers, and the percentage and absolute numbers of CD4+, CD25+, CD127, FOXP3+presented no significant difference $(\mathrm{P}=1.168$, $\mathrm{P}=1.119$, respectively).

According to the expression of CD45RA Treg can divided to subset CD4+CD25+CD127F OXP $3+$ C D 45 R A +, C D 4 + CD $25+$ CD 127 FOXP3+CD45RA- A comparison of the percentage and absolute numbers reveals no significant difference $(\mathrm{P}=1.777, \mathrm{P}=1.679, \mathrm{P}=1.286, \mathrm{P}=1.24)$. Likewise, comparing the FOXP3 MFI among sulfur mustard exposed and healthy control confirmed no significant difference $(\mathrm{P}=0.527)$. There is no correlation between spyrometric parameters and Treg frequency, as shown in (Table 3).

Additionally, as the demographic and clinical characteristics of the population (Table 1) demosntrates, there is a significant difference $(\mathrm{P}=0.03)$ in age between the two groups. To adjust for age's confounding effect, ANCOVA (Analysis of Covariance) was employed. Consequently, similar results were obtained.

\section{Discussion}

Concerning disturbed immune system balance and the risk of malignancy in sulfur mustard exposed patients, evaluating Treg cells' frequency and other aspects 
Table 1. The baseline characteristics of the research subjects

\begin{tabular}{|c|c|c|c|c|}
\hline \multirow{2}{*}{ Characteristics } & \multicolumn{2}{|c|}{ Mean $\pm S D$} & \multirow{2}{*}{$\mathbf{P}^{*}$} & \multirow{2}{*}{ Adjusted by Age P (ANCOVA)** } \\
\hline & Control Group ( $n=12$ ) & Exposed Group (n=12) & & \\
\hline Age $\left(y^{a}\right)$ & $41.83 \pm 7.67$ & $53.09 \pm 3.08$ & - & - \\
\hline BMI $\left(\mathrm{kg} / \mathrm{m}^{2}\right)$ & $27.86 \pm 2.93$ & $28.62 \pm 5.21$ & 0.660 & - \\
\hline FVC & - & $74.0 \pm 16.4$ & - & - \\
\hline FEV1 & - & $74.09 \pm 16.39$ & - & - \\
\hline FEV1/FVC & - & $104.73 \pm 14.19$ & - & - \\
\hline $\mathrm{WBC}\left(10^{3} / \mathrm{mL}\right)$ & $6.56 \pm 1.25$ & $7.32 \pm 1.78$ & 0.237 & 0.379 \\
\hline Lymphocyte $\left(10^{3} / \mathrm{mL}\right)$ & $2.31 \pm 0.58$ & $2.61 \pm 1.03$ & 0.377 & 0.389 \\
\hline Monocyte $\left(10^{3} / \mathrm{mL}\right)$ & $0.55 \pm 0.16$ & $0.54 \pm 0.14$ & 0.885 & 0.346 \\
\hline Basophil $\left(10^{3} / \mathrm{mL}\right)$ & $0.03 \pm 0.02$ & $0.03 \pm 0.02$ & 0.619 & 0.637 \\
\hline Eosinophil $\left(10^{3} / \mathrm{mL}\right)$ & $0.19 \pm 0.1$ & $0.23 \pm 0.12$ & 0.384 & 0.941 \\
\hline Neutrophil $\left(10^{3} / \mathrm{mL}\right)$ & $3.48 \pm 0.86$ & $3.92 \pm 1.01$ & 0.262 & 0.403 \\
\hline
\end{tabular}

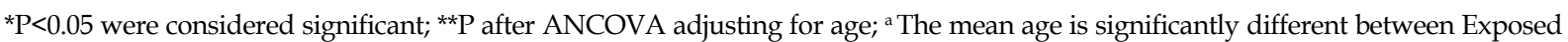
and Control groups. BMI: Body Mass Index; FVC: Forced Vital Capacity; FEV1: Forced; Expiratory Volume in one second; WBC: White Blood Cell; SD: Standard Deviation.

of immune response can shed light on monitoring injury development and prevention $[9,11,12,23]$. In the present study, we first investigated the percentage and the absolute number of CD4+cells. We found no significant change in the frequency of Treg cells by calculating both absolute counts and percentages in different definitions, emphasizing the Treg subset. CD4+CD25+CD127- and CD4+CD25+CD127-FOXP3+frequencies were not different in victims of chemical weapons compared to healthy volunteers.

The frequency of CD45RA+Treg cells as effector subset and CD45RA-Treg cells as naïve subset were studied in patients; however, no significant difference was observed compared to the control group. Furthermore, we show that FOXP3 MFI among sulfur mustard exposed patients and healthy control members have undergone no significant change.

In contrast to our findings, one study by Imani et al. demonstrated that CD4+, FOXP3+cells increased in sulfur mustard exposed patients as Treg [17]. In COPD patients, whose symptoms and immune system status share much in common with sulfur mustard exposed patients, some studies, such as Paats et al., indicate no changes in $\mathrm{CD} 4+, \mathrm{FOXP} 3+, \mathrm{CD} 25+$ frequencies [24]. Some of them, such as Kalathil et al., indicated an increase in Treg cell frequency but with a defect in effector T-cell in the vicinity of Treg, using an in vitro suppression assay [25].

Controversial results between studies might be explained by the variety of Treg definitions between studies, differences in laboratory methods for examining these cells, and differences in staging and stages of chemical weapon victims' illness. This study included patients with mild to moderate lung complications because we considered this critical stage and its changes to be helpful to clinicians for taking action against and preventing the development of severe stages. Despite the mentioned inconsistency, we can state that the inflammatory arm of the immune system overwhelms the regulatory and anti-inflammatory arms, and to confirm this claim, Treg function should be taken into account in future research. We used both $\mathrm{CBC}$ and flow cytometry to measure the absolute cell number in this study. We recommend performing single-platform tests by using count beads in flow cytometry.

There is no correlation between spyrometric parameters and Treg cell frequency. The lack of correlation may be due to the detection of Treg in blood. Examining Treg in the site of lungs or lavage would be a better option. 
Table 2. Frequency of lymphocytes, CD4+T cell, and Treg in sulfur mustard exposed patients and healthy controls

\begin{tabular}{|c|c|c|c|c|}
\hline \multirow{2}{*}{ Variables } & \multicolumn{2}{|c|}{ Mean $\pm S D$} & \multirow{2}{*}{$\mathbf{P}^{*}$} & \multirow{2}{*}{$\begin{array}{c}\text { Adjusted } \\
\text { by Age } \mathrm{P} \\
(\mathrm{ANCOVA})^{* *}\end{array}$} \\
\hline & Control Group ( $n=12)$ & Exposed Group ( $n=12)$ & & \\
\hline CD4+cells (cells/ $\mu \mathrm{L}$ ) & $970.34 \pm 354.79$ & $951.42 \pm 380.71$ & 0.901 & 0.815 \\
\hline CD4+cells (\% lymphocytes) & $41.29 \pm 8.18$ & $36.31 \pm 5.25$ & 0.09 & 0.216 \\
\hline CD4+CD25+CD127-Treg cells (cells $/ \mu \mathrm{L})$ & $90.2 \pm 59.95$ & $123.08 \pm 98.26$ & 0.333 & 0.191 \\
\hline CD4+CD25+CD127-Treg cells (\% lymphocytes) & $3.74 \pm 2.02$ & $4.38 \pm 1.69$ & 0.413 & 0.091 \\
\hline CD4+CD25+CD127-, FOXP3+Treg cells (cells/ $\mu \mathrm{L}$ ) & $34.6 \pm 24.35$ & $37.95 \pm 16.33$ & 0.696 & 0.209 \\
\hline CD4+CD25+CD127-, FOXP3+Treg cells (\% lymphocytes) & $1.42 \pm 0.83$ & $1.51 \pm 0.47$ & 0.76 & 0.268 \\
\hline CD4+,CD25+,CD127-,FOXP3+,CD45RA+Treg cells (cells/ $\mu \mathrm{L})$ & $12.3 \pm 9.72$ & $8.56 \pm 5.56$ & 0.263 & 0.679 \\
\hline CD4+,CD25+,CD127-,FOXP3+,CD45RA+Treg cells (\% lymphocytes) & $0.5 \pm 0.34$ & $0.36 \pm 0.23$ & 0.239 & 0.777 \\
\hline CD4+,CD25+,CD127-,FOXP3+,CD45RA-Treg cells (cells/ $\mu \mathrm{L})$ & $23.17 \pm 15.34$ & $30.03 \pm 14.46$ & 0.272 & 0.14 \\
\hline CD4+,CD25+,CD127-,FOXP3+,CD45RA-Treg cells (\% lymphocytes) & $0.97 \pm 0.52$ & - & 0.313 & 0.186 \\
\hline MFI of FOXP3 in CD4+CD25+CD127low FOXP3+Treg cells & $7046.75 \pm 1659.849$ & $7691.33 \pm 1168.28$ & 0.283 & 0.527 \\
\hline
\end{tabular}

${ }^{*} \mathrm{P}<0.05$ was considered significant; ${ }^{*} \mathrm{P}$ after ANCOVA adjusting for age; WBC: White Blood Cell; SD: Standard Deviation; MFI: Mean Floursent Intensity.

Table 3. Correlation between spirometric parameters and Treg frequency

\begin{tabular}{|c|c|c|c|c|c|c|}
\hline \multirow{2}{*}{ Variables } & \multicolumn{2}{|c|}{ FVC } & \multicolumn{2}{|c|}{ FEV1 } & \multicolumn{2}{|c|}{ FEV1/FVC } \\
\hline & $\mathbf{P}$ & $\mathbf{r}$ & $\mathbf{P}$ & $\mathbf{r}$ & $\mathbf{P}$ & $\mathbf{r}$ \\
\hline CD4+cells (cells/ $\mu \mathrm{L}$ ) & 0.976 & 0.01 & 0.89 & -0.47 & 0.833 & 0.72 \\
\hline CD4+cells (\% lymphocytes) & 0.851 & 0.64 & -0.679 & -0.141 & 0.183 & -0.433 \\
\hline CD4+CD25+CD127-Treg cells (cells $/ \mu \mathrm{L}$ ) & 0.957 & -0.18 & 0.933 & 0.29 & 0.760 & 0.104 \\
\hline CD4+CD25+CD127-Treg cells (\% lymphocytes) & 0.783 & 0.094 & 0.704 & 0.13 & 0.846 & 0.059 \\
\hline CD4+CD25+CD127-,FOXP3+Treg cells (cells/ $\mu \mathrm{L})$ & 0.893 & 0.46 & 0.893 & 0.46 & 0.838 & 0.7 \\
\hline CD4+CD25+CD127-,FOXP3+Treg cells (\% lymphocytes) & 0.825 & 0.076 & 0.857 & 0.062 & 0.987 & 0.005 \\
\hline CD4+,CD25+,CD127-,FOXP3+,CD45RA+Treg cells (cells/ $\mu \mathrm{L})$ & 0.904 & 0.41 & 0.941 & 0.25 & 0.913 & -0.38 \\
\hline CD4+,CD25+,CD127-,FOXP3+,CD45RA+Treg cells (\% lymphocytes) & 0.658 & 0.151 & 0.714 & 0.125 & 0.784 & -0.94 \\
\hline CD4+,CD25+,CD127-,FOXP3+,CD45RA-Treg cells (cells/ $\mu \mathrm{L})$ & 0.936 & 0.27 & 0.892 & 0.47 & 0.731 & 0.117 \\
\hline CD4+,CD25+,CD127-,FOXP3+,CD45RA-Treg cells (\% lymphocytes) & 0.966 & -0.14 & 0.992 & 0.3 & 0.770 & 0.1 \\
\hline
\end{tabular}


A
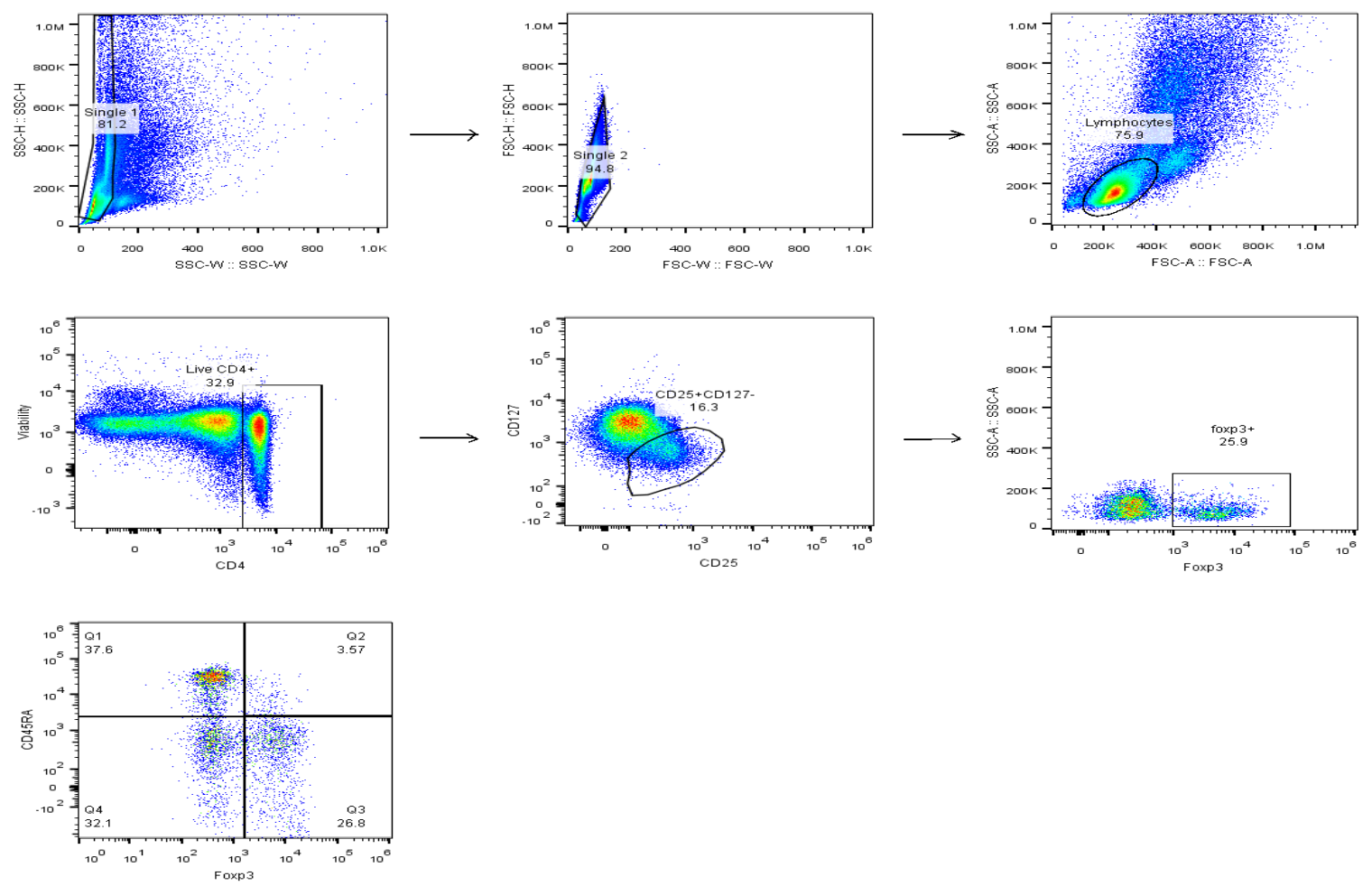

B
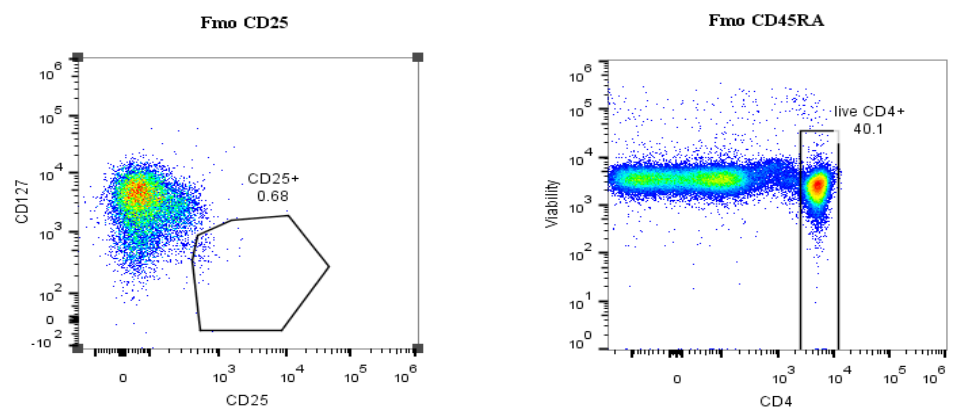

C

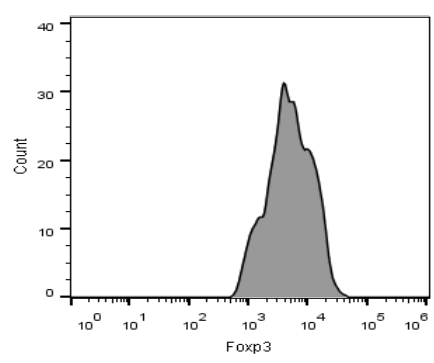

ImimoRegulation

Figure 1. The flowcytometry analysis of Treg cells

Left to right: A: PBMC were initially gated based on SSC-H versus SSC-W and FSC-H versus FSC-W to exclude doublet cells. Next, The lymphocytes gate was determined by an FSC-A versus side-scatter area (SSC-A) plot. then CD25 was plotted against CD127 in CD4+T live lymphocytes and CD25+CD127- based on FOXP3 and CD45RA divided into 2 subtype CD4+CD25+CD127-FOXP3+CD45RA+and CD4+CD25+CD127- FOXP3+CD45RA- and based on FOXP3 and SSC-A into CD4+CD25+CD127- FOXP3+and CD4+CD25+CD127FOXP3; B: FMO (fluorescence minus one) control prepared to determine correct gating for CD25 and CD45; C) FOXP3 expression level in CD4+CD25+CD127-FOXP3+gated Treg cell. 


\section{Conclusion}

The frequency of Treg cells revealeds no significant difference between patients exposed to sulfur mustard, who suffer from mild pulmonary complications, and healthy volunteers. To better understand the role of these cells in pulmonary complications of the patients, further studies are necessary.

\section{Ethical Considerations}

\section{Compliance with ethical guidelines}

This study was approved by the Local Ethics Committee of Shahed University (Code:REC.13961218). Informed consent was obtained from all participants.

\section{Funding}

This research did not receive any grant from funding agencies in the public, commercial, or non-profit sectors.

\section{Authors' contributions}

Conceptualization, supervision: Tooba Ghazanfari; Investigation: Alireza Sabetpour; Methodology, statistic analysis, writing - original draft: Nafiseh Zand, Ensieh Sadat Mirsharif, Mahmood Bozorgmehr, and Mohammad Mahdi Naghizade; Writing - review \& editing: All authors.

\section{Conflicts of interest}

The authors declared no conflict of interest.

\section{Acknowledgments}

We would like to express our greatfulness to the support of the Immunoregulation Research Center.

\section{References}

[1] Emami MH, Talaei M, Panahi Y, Saburi A, Ghanei M. Efficacy of omeprazole on cough, pulmonary function and quality of life of patients with sulfur mustard lung injury: A placebo-control, cross-over clinical trial study. Journal of Research in Medical Sciences. 2014; 19(11):1027-33. [PMID] [PMCID]

[2] Borak J, Sidell FR. Agents of chemical warfare: Sulfur mustard. Annals of Emergency Medicine. 1992; 21(3):303-8. [DOI:10.1016/S0196-0644(05)80892-3]

[3] Benschop HP, van der Schans GP, Noort D, Fidder A, MarsGroenendijk RH, de Jong LP. Verification of exposure to sul- fur mustard in two casualties of the Iran-Iraq conflict. Journal of Analytical Toxicology. 1997; 21(4):249-51. [DOI:10.1093/ jat/21.4.249] [PMID]

[4] Holisaz M, Raigany S, Hafezy R, Bakhshandeh H. The role of chemical warfare agents in inducing peripheral neuropathy. Kowsar Medical Journal. 2003; 8:39-46. https://scholar. google.com/scholar?hl=en\&as_sdt $=0 \% 2 \mathrm{C} 5 \& \mathrm{q}=+$ The + role +

[5] Hassan ZM, Ebtekar M. Modeling for immunosupression by sulfur mustard. International Immunopharmacology. 2001; 1(3):605-10. [DOI:10.1016/S1567-5769(00)00036-9] [PMID]

[6] Hefazi M, Attaran D, Mahmoudi M, Balali-Mood M. Late respiratory complications of mustard gas poisoning in Iranian veterans. Inhalation Toxicology. 2005; 17(11):587-92. [DOI:10.1080/08958370591000591] [PMID]

[7] Razavi SM, Salamati P, Saghafinia M, Abdollahi M. A review on delayed toxic effects of sulfur mustard in Iranian veterans. Daru: Journal of Faculty of Pharmacy, Tehran University of Medical Sciences. 2012; 20(1):51. [DOI:10.1186/2008-2231-2051] [PMID] [PMCID]

[8] Khateri S, Ghanei M, Keshavarz S, Soroush M, Haines D. Incidence of lung, eye, and skin lesions as late complications in 34,000 Iranians with wartime exposure to mustard agent. Journal of Occupational and Environmental Medicine. 2003; 45(11):1136-43. [DOI:10.1097/01.jom.0000094993.20914.d1] [PMID]

[9] Ghanei M, Adibi I. Clinical review of mustard lung. Iranian Journal of Medical Sciences. 2007; 32(2):58-65. https://ijms. sums.ac.ir/article_39925.html

[10] Ghassemi-Broumand M, Aslani J, Emadi S-N. Delayed ocular, pulmonary, and cutaneous complications of mustards in patients in the City of Sardasht, Iran. Cutaneous and Ocular Toxicology. 2008; 27(4):295-305. [DOI:10.1080/15569520802327807] [PMID]

[11] Hosseini-khalili A, Haines DD, Modirian E, Soroush M Khateri S, Joshi R, et al. Mustard gas exposure and carcinogenesis of lung. Mutation Research. 2009; 678(1):1-6 [DOI:10.1016/j.mrgentox.2009.05.022] [PMID] [PMCID]

[12] Hassan ZM, Ebtekar M, Ghanei M, Taghikhani M, Daloii MRN, Ghazanfari T. Immunobiological consequences of sulfur mustard contamination. Iranian Journal of Allergy, Asthma and Immunology. 2006; 5(3):101-8. [PMID]

[13] Imani S, Salimian J, Fu J, Ghanei M, Panahi Y. Th17/Tregrelated cytokine imbalance in sulfur mustard exposed and stable Chronic Obstructive Pulmonary (COPD) patients: Correlation with disease activity. Immunopharmacology and Immunotoxicology. 2016; 38(4):270-80. [DOI:10.1080/0 8923973.2016.1188402] [PMID]

[14] Emad A, Emad Y. Levels of cytokine in Bronchoalveolar Lavage (BAL) fluid in patients with pulmonary fibrosis due to sulfur mustard gas inhalation. Journal of Interferon \& Cytokine Research. 2007; 27(1):38-43. [DOI:10.1089/ jir.2006.0084] [PMID]

[15] Ghazanfari T, Kariminia A, Yaraee R, Faghihzadeh S, Ardestani SK, Ebtekar M, et al. Long term impact of sulfur mustard exposure on peripheral blood mononuclear subpopulations-Sardasht-Iran Cohort Study (SICS). International Immunopharmacology. 2013; 17(3):931-5. [DOI:10.1016/j. intimp.2012.12.023] [PMID] 
[16] Shaker Z, Hassan Z, Sohrabpoor H, Mosaffa N. The immunostatus of $\mathrm{T}$ helper and $\mathrm{T}$ cytotoxic cells in the patients ten years after exposure to sulfur mustard. Immunopharmacology and Immunotoxicology. 2003; 25(3):423-30. [DOI:10.1081/IPH-120024509] [PMID]

[17] Imani S, Salimian J, Bozorgmehr M, Vahedi E, Ghazvini A, Ghanei M, et al. Assessment of Treg/Th17 axis role in immunopathogenesis of chronic injuries of mustard lung disease. Journal of Receptors and Signal Transduction. 2016; 36(5):531-41. [DOI:10.3109/10799893.2016.1141953] [PMID]

[18] Yamaguchi T, Wing JB, Sakaguchi S. Two modes of immune suppression by Foxp3+regulatory T cells under inflammatory or non-inflammatory conditions. Seminars in Immunology. 2011; 23(6):424-30. [DOI:10.1016/j.smim.2011.10.002] [PMID]

[19] Askenasy N, Kaminitz A, Yarkoni S. Mechanisms of T regulatory cell function. Autoimmunity Reviews. 2008; 7(5):370-5 [DOI:10.1016/j.autrev.2008.03.001] [PMID]

[20] Singer BD, King LS, D'Alessio FR. Regulatory T cells as immunotherapy. Frontiers in Immunology. 2014; 5:46. [DOI:10.3389/fimmu.2014.00046] [PMID] [PMCID]

[21] Santegoets SJ, Dijkgraaf EM, Battaglia A, Beckhove P, Britten $\mathrm{CM}$, Gallimore A, et al. Monitoring regulatory T cells in clinical samples: Consensus on an essential marker set and gating strategy for regulatory $\mathrm{T}$ cell analysis by flow cytometry. Cancer Immunology, Immunotherapy. 2015; 64(10):1271-86. [DOI:10.1007/s00262-015-1729-x] [PMID] [PMCID]

[22] Crapo RO, Hankinson JL, Irvin C, MacIntyre NR, Voter KZ, Wise RA, et al. Graham Standardization of Spirometry, 1994 Update. American Thoracic Society. American Journal of Respiratory and Critical Care Medicine. 1995; 152(3):1107-36. [DOI:10.1164/ajrccm.152.3.7663792] [PMID]

[23] Kehe K, Szinicz L. Medical aspects of sulphur mustard poisoning. Toxicology. 2005; 214(3):198-209. [DOI:10.1016/j. tox.2005.06.014] [PMID]

[24] Paats MS, Bergen IM, Hoogsteden HC, van der Eerden MM, Hendriks RW. Systemic CD4+ and CD8+T cell cytokine profiles correlate with GOLD stage in stable COPD. European Respiratory Journal. 2012; 40(2):330-7. [DOI:10.1183/09031936.00079611] [PMID]

[25] Kalathil SG, Lugade AA, Pradhan V, Miller A, Parameswaran GI, Sethi S, et al. T-regulatory cells and programmed death $1+\mathrm{T}$ cells contribute to effector T-cell dysfunction in patients with chronic obstructive pulmonary disease. American Journal of Respiratory and Critical Care Medicine. 2014; 190(1):40-50. [DOI:10.1164/rccm.2013122293OC] [PMID] [PMCID] 